\title{
Kernos
}

Revue internationale et pluridisciplinaire de religion grecque antique

15 | 2002

Varia

\section{Alain LERNOULD, Physique et théologie. Lecture du Timée de Platon par Proclus}

\section{Carine Van Liefferinge}

\section{OpenEdition \\ Journals}

\section{Édition électronique}

URL : http://journals.openedition.org/kernos/1454

DOI : 10.4000/kernos. 1454

ISSN : 2034-7871

\section{Éditeur}

Centre international d'étude de la religion grecque antique

\section{Édition imprimée}

Date de publication : 1 janvier 2002

ISSN : 0776-3824

\section{Référence électronique}

Carine Van Liefferinge, «Alain LERnould, Physique et théologie. Lecture du Timée de Platon par Proclus », Kernos [En ligne], 15 | 2002, mis en ligne le 22 avril 2011, consulté le 24 septembre 2020. URL : http://journals.openedition.org/kernos/1454 ; DOI : https://doi.org/10.4000/kernos.1454 
philologie classique, l'A. use donc d'une méthode rigoureuse, sait analyser les textes et ordonner ses exposés avec clarté autour des thématiques propres à chaque auteur. Il est certes des points particuliers que l'on aimerait discuter, mais le principal reproche que l'on pourrait faire à l'ouvrage, me semble-t-il, c'est de manquer d'une solide introduction qui eût permis à l'A. de nouer sa problématique en précisant aussi sa terminologie ( $c f$. mes remarques initiales), d'exposer également sa démarche et de justifier certains choix effectués durant son long parcours. S'agissant de ce dernier point, je prendrai deux exemples. Chez les présocratiques, on veut bien admettre que l'unique fragment conservé d'Anaximandre ait droit à une attention particulière, compte tenu de ses implications théologiques possibles, mais on s'étonne que n'apparaisse nulle part le nom de Xénophane, en qui pourtant de nombreux fragments font voir un véritable théologien et qui a grandement pesé, à tout le moins indirectement, sur le développement d'une théodicée en dénonçant les impasses d'un anthropomorphisme qui attribue aux dieux les pires comportements des hommes. Nul, d'autre part, ne déplorera qu'un chapitre final soit réservé à un penseur chrétien, écrivant en latin, de la taille d'un saint Augustin, mais, que je sache, il n'est pas le premier penseur chrétien, tant en Orient qu'en Occident, à avoir réfléchi sur le sujet. Le fil historique est donc ici partiellement coupé; il eût été convenant à tout le moins d'en avertir le lecteur et de s'en expliquer.

Ces critiques mineures n'enlèvent rien à l'intérêt que, d'un bout à l'autre, on prend à la lecture de cette étude que recommande aussi une présentation soignée.

\section{A. Motte}

(Université de Liège)

Alain Lernould, Physique et théologie. Lecture du Timée de Platon par Proclus, Lille, Presses Universitaires du Septentrion, 2001. 1 vol. $16 \times 24 \mathrm{~cm}$, 405 p. (Problématiques pbilosopbiques). ISBN : 2-85939-644-6.

Version remaniée d'une thèse soutenue à Lille en 1992, cet ouvrage relance la question du pythagorisme du Timée de Platon, question qui fut l'objet de controverses dès l'Antiquité. Ainsi, pour le philosophe néoplatonicien Proclus, l'inspiration pythagoricienne du Timée ne fait aucun doute. L'A. entreprend précisément de montrer comment la volonté de Proclus d'attribuer un caractère pythagoricien à cette cuvre de Platon amène le Diadoque à procéder dans son Commentaire à un profond réaménagement du dialogue commenté. L'ouvrage comporte deux grandes parties. La première partie, elle-même divisée en quatre sections suivies de conclusions, étudie le but (scopos) du Timée tel qu'il est assigné par Proclus au dialogue platonicien, procède à une analyse du Commentaire dans son ensemble, met en lumière le découpage du dialogue par Proclus et les raisons qui le sous-tendent. Afin de comprendre l'arrière-plan du Commentaire, l'A. rappelle prélablement la hiérarchie des êtres selon Proclus et l'ordre de lecture des dialogues de Platon dans l'École d'Athènes du ve $s$. Le but du Timée selon Proclus est de s'attacher à la physique dans sa totalité, mais les différents moments de la création correspondent selon lui aux étapes de la remontée vers les causes divines du monde : la physique devient ainsi une sorte de théologie. De l'analyse d'ensemble du Commentaire, suivie de la présentation de la divisio textus établie par Proclus, il apparaît d'une part une parfaite concordance entre le programme que s'est fixé Proclus dans son introduction et le plan dégagé de l'ensemble du traité, d'autre part une inadéquation entre les divisions de la démiurgie dans l'In Timaeum et le plan dialectique en trois parties adopté par Platon. Le Timée de Platon ainsi recomposé et restructuré par le commentateur, 
l'A. distingue dès lors le «Timée de Platon » et le «Timée de Proclus ». Ce dernier apparaît surtout comme ayant escamoté la seconde partie du Timée et réduit celui-ci à sa première partie. Cherchant les raisons de ces écarts, l'A. conclut cette première partie en montrant que Proclus a cherché à concilier le discours de Timée, qui partant des premiers principes s'en éloigne peu à peu pour s'enfoncer dans la matière, avec la volonté, caractéristique du Pythagorisme en physique, de toujours remonter aux causes premières : en réalité, le «Timée de Proclus " est dans son ensemble un traité des causes premières du monde. La seconde partie de l'ouvrage vise à mettre en évidence, par un commentaire du Commentaire, la progression dialectique que Proclus imprime à ce dernier. Elle comporte trois sections précédées de préalables précisant le vocabulaire mathématique utilisé par Proclus et la classification des sciences selon ce dernier. Peut alors commencer l'analyse détaillée du texte de Proclus de laquelle ressort la thèse défendue par l'A., à savoir que l'exposé physique de Timée se déploie "dialectiquement ", c'est-à-dire selon le mode propre à la théologie, science première. Les première et deuxième sections abordent la façon dont Proclus repose le problème fondamental de la physique, "le monde est-il né ou pas ? ». Elles passent en revue en les mettant en rapport, l'une les différentes «hypothèses", l'autre les "démonstrations » de Platon, telles qu'elles sont envisagées par Proclus de manière à aboutir à la thèse unique selon laquelle le monde est absolument devenu en tant que possédant son essence dans le temps. L'A. souligne avec pertinence un fait important dans la pratique du commentaire en général : pour aboutir à ses conclusions, l'auteur du commentaire substitue ses propres catégories, par exemple celles du devenu et de l'éternel, à celles de l'auteur commenté. Dans sa lecture des prémisses du Timée, vues par Proclus comme des hypothèses préparant les démonstrations, le philosophe néoplatonicien préconise une lecture en profondeur en vertu de laquelle les hypothèses constituent une première remontée et les démonstrations, dont elles sont les préalables, une seconde remontée dialectique vers les Causes du Monde. La troisième section envisage enfin le traité de la Démiurgie du Monde comme troisième remontée dialectique. En mettant en lumière la progression que Proclus imprime entre ces trois découvertes des Causes du Monde, l'A. dévoile ainsi un élément essentiel pour la compréhension du Commentaire de Proclus qui nous ramène à la question de pythagorisme du Timée : la physique "pythagoricienne ", c'est-à-dire théologique, de Platon, se caractérise, selon Proclus, par le dépassement de l'exposé géométrique et son élévation à un niveau dialectique au sens néoplatonicien du terme, en tant que préparation à la saisie intellectuelle des causes premières.

- Carine Van Liefferinge (Université libre de Bruxelles)

Bernard Pouderon, Yves-Marie Duval (éds), L'Historiographie de l'Église des premiers siècles, Paris, Beauchesne, 2001. 1 vol. $13,5 \times 21,5 \mathrm{~cm}, \mathrm{xv}+586 \mathrm{p}$. (Théologie bistorique, 114). ISBN : 2-7010-1413-1.

Ce beau livre contient les Actes du Second Colloque international d'Études patristiques, qui a eu lieu à Tours du 11 au 13 septembre 2000 et a été organisé par l'Université de Tours et l'Institut Catholique de Paris. Il ne témoigne pas seulement du renouveau des études patristiques en France, mais également de la popularité dont le thème du colloque jouit aujourd'hui. En effet, la bibliographie centrée sur l'historiographie de l'Église ancienne devient de plus en plus vaste: les éditions critiques se multiplient, des traductions en langues modernes ont vu le jour, de multiples études ont paru, en France comme à l'étranger, et les érudits échangent leurs connaissances lors de nombreux congrès. Citons ici, à titre 